\title{
Safety Training Monitoring System of Ski Resort Based on Wireless Sensor Network
}

\author{
Zhidong Lin, Yanmin Fu, and Xuefeng Deng $\mathbb{D}$ \\ Physical Education and Training, Harbin Normal University, Harbin 150000, Heilongjiang, China \\ Correspondence should be addressed to Xuefeng Deng; dxf1999@hrbnu.edu.cn
}

Received 14 December 2021; Revised 13 January 2022; Accepted 20 January 2022; Published 17 February 2022

Academic Editor: Deepak Kumar Jain

Copyright ( $\odot 2022$ Zhidong Lin et al. This is an open access article distributed under the Creative Commons Attribution License, which permits unrestricted use, distribution, and reproduction in any medium, provided the original work is properly cited.

\begin{abstract}
With the gradual rise in China's ski industry, skiing is now the most common sport among winter outdoor sports, which is closely related to the origin of safety issues. Based on the ski resort, training is the key to safety detection, only to ensure the safety of training personnel and to maintain the momentum of high-speed development of skiing sports for a long time. The monitoring system plays an important role in the wireless sensor network monitoring system. Common monitoring systems are used for agriculture, smart home, and outdoor environment monitoring systems, most of which are professional fields. Based on the safety of ski resorts, this paper puts forward the research of ski training safety system based on the wireless sensor. In the research of ski training detection system based on the wireless sensor network, first, the safety system of domestic ski resort is analyzed and detailed suggestions are given. Then, this paper studies the routing protocol of wireless communication technology in detail and compares the service life and energy consumption of nodes under the use of two different routing protocols in wireless sensor networks. According to the data experiment in this paper, LEACH-MH protocol has great advantages over the LEACH protocol. The LEACH-MH protocol detects 185 more rounds than the LEACH protocol in the total detection lifetime. In the detection of energy consumption of $100 \mathrm{~m} * 100 \mathrm{~m}$, the LEACH protocol consumes 50J in 400 rounds, while the LEACH-MH protocol consumes only $50 \mathrm{~J}$ in 760 rounds.
\end{abstract}

\section{Introduction}

During skiing training, because the speed is too fast, it will accidentally hit other people or other obstacles. Due to the remote location and large area of the ski resort, it is difficult to treat the injured in the first place. Therefore, it is necessary to establish a ski resort safety training detection system to ensure the safety of ski resort tourists and training personnel.

Many scholars have studied the application of wireless sensor and achieved good results. For example, Abdul Latiff proposed a rider training monitoring system based on wireless sensor networks. A stable and reliable wireless rider monitoring system is very important for the establishment of an intelligent and efficient sport management program. He has developed and tested the training monitoring system in a real cycling training environment at a velodrome. The system is designed to minimize packet loss. Based on TelG nodes, he developed customized sensor nodes as forwarding nodes and relay nodes, forming WSN. The WSN links to a cloud network over the Internet, then builds the cloud network, and designs end-user applications for data access. It has carried out many experiments in real-life scenarios of racing tracks to measure the reliability of the system architecture. However, based on the actual experiment, the sensor will be damaged in the process of intense experiment and cannot be tested multiple times [1]. Zhou proposed a safety monitoring system based on an information physics system for blind cranes in the subway and underground buildings. For his example study, he chose the Wuhan subway tunnel that crosses China's Yangtze River. He designed a mud shield machine with a tool diameter of $15.76 \mathrm{~m}$, which can lift $44 \mathrm{~m}$ in underground tunnel shafts. To overcome this difficulty, CPS-SMS was brought forward to simulate and monitor the lifting process to avoid unsafe conditions of the crane and the cutter wheel being lifted. By using IoT technologies such as positioning and tracking based on wireless sensors, self-organizing $\mathrm{Wi}-\mathrm{Fi}$, and two- 
way communication, it can prevent accidents in the process of changing lifting, and on this basis, no collision accidents and casualties have occurred. Practitioners can apply CPSSMS to a variety of construction projects to reduce the likelihood of blind lifting safety risks in complex environments. However, the system is too professional to be used by beginners [2]. Niu has implemented a wireless monitoring system for heavy metals in water based on improved optical measurement and wireless sensor network technology. The modular design method analyzes the characteristics of optical sensor unit and equipment and develops the hardware design and embedded software design of optical wireless sensor node for the system. Finally, he evaluated the performance of the system. Although the system has the characteristics of high precision, low cost, and low power consumption, the interface of the system is not humanized enough [3]. Lutakamale and Kaijage proposed a wildfire monitoring and detection system based on wireless sensor networks. The system detects fires by monitoring ambient temperature, humidity, and smoke. As soon as a fire is detected, a warning message containing the possible location of the fire is immediately sent to the responsible agency over the cellular network. To make the system even more effective, communities living near forests or national parks can use cell phones to send warning messages to responsible authorities through the same system if they witness a wildfire or illegal activity. To make the system fully functional, the only requirement is to provide cellular coverage in forests or national parks to enable SMS services. The prototype system was developed using an Arduino microcontroller, multiple sensors to detect temperature, relative humidity and smoke, and wireless network connectivity modules. However, according to the experimental data, he adopted cellular network coverage, which was too expensive compared with other detection methods [4]. In order to realize the collection, management, and visualization of real-time information of farmland, Zhang Xiao proposed a node for the automatic collection of information based on solar panels. This kind of node is composed of an information sensor, STM32 MCU, LCD, GPRS module, automatic collection solar module, and monitoring interface. Then, based on the data collected by the sensor, the analyzed data are displayed on the LCD and then sent to the host. In actual operation, the node turns on the power consumption mode, so as to achieve reasonable use of energy. According to the results, this kind of node can accurately transmit data, and the power supply module can meet the use of rainy days for 5 days, but the data collection based on this experiment is too tedious [5].

Hammoudeh pointed out that the security of the border is of vital importance to every country, and changes in the border can surely bring new challenges and intensify friction between countries. The wireless sensing network is a low-cost monitoring network that can provide corresponding solutions for monitoring large and complex scenes. The linear network topology analyzed based on the scene of the monitoring area poses new challenges. In the article, first, an indicator is defined to measure the standard of WSN transmission detection. In addition, a method has been developed to calculate the number of nodes and maintain wireless connections within the network. Then, a new cross-layer routing protocol called hierarchical partition graph (LDG) is proposed, which is specifically used to solve the communication requirements and link reliability of topological linear WSN applications. The performance of the protocol proposed in his paper is not mainstream usage protocol in reality [6].

Wang proposed that wireless sensing networks can be constructed and monitored system automation through a variety of methods. One emerging app is to guide drivers quickly to empty spaces in large parking lots during rush hour. Through continuous information and gradient ascent methods, he proposed a high-line-rate parking navigation system. Theoretical analysis proves the convergence of the algorithm, which can effectively prevent parking lot congestion caused by parking. According to research, this algorithm is more efficient than existing algorithms. However, this navigation value is applicable to traditional parking lots, not the latest elevator parking lots [7].

With more and more problems related to ski safety, it is difficult to apply the development stages of skiing to different parts of the country. At present, there are few studies on safety inspection of ski resorts in China, especially on safety training inspection of ski resorts. Aiming at the safety dilemma of the ski resort, the detection of the ski resort area by the wireless sensor can provide timely help for tourists and other skiers and improve the safety factor of the ski resort. This paper provides intelligent and real-time detection measures for ski resorts to improve the safety of ski resort personnel [8].

\section{Wireless Sensor Detection System of Ski Resorts}

2.1. Ski Resort Safety Measures. Ordinary tourists pay more attention to the entertainment of ski resorts. They seldom consider the safety of ski resorts when choosing ski resorts and do not consider the safety facilities of ski resorts as an important basis for choosing ski resorts. In terms of infrastructure, the safety problems often encountered are as follows: some ski resorts do not clearly indicate the grade, length, and slope of the ski slopes, which makes it inconvenient for skiers to choose the slopes according to their own level and avoid risks; the settings of the ski slope protection net are uneven, some dangerous areas lack protective nets, some do not meet the specifications, some are neglected in daily maintenance, and there are certain hidden dangers; some ski slopes have a snow layer thickness of less than 0.3 meters, and some places have exposed debris such as soil and rocks. The construction of safety facilities is the most basic measure to ensure the safety of skiing participants. If the construction of safety facilities is ignored, potential safety risks will be buried [9]. Therefore, the safety management of the ski resort mainly has the following suggestions.

2.1.1. Improving the Market Supervision System. The government should strengthen the control of ski resorts, clarify the scope of responsibility, establish a safety certification 
system for ski resorts, and collect requirements and evaluation indicators from multiple departments, so as to realize multifield joint inspection and supervision improve the supervision level and reflect the fairness of supervision [10].

\subsubsection{Strengthening the Safety Management of the Ski Resort.} While standardizing its rules and regulations, each ski resort should define the person in charge of each link to take charge of specific tasks and make full and reasonable use of favorable resources [11].

2.1.3. Improving the Construction of Ski Facilities. At the beginning of the construction of the ski resort, the site selection should be reasonable in view of the safety problems, and the multidimensional consideration should be carried out to eliminate the safety hidden dangers from the source. In addition, actively improving the conditions of equipment and facilities can greatly improve the skiing experience of participants, and the safety is fully guaranteed [12].

2.1.4. Focusing the Cultivation of Talents. The development of domestic skiing is relatively late, and people do not know much about the dangers of skiing. Therefore, skiing professionals should play a more important role in public participation in skiing.

2.1.5. Improving the Emergency Rescue Capacity of the Ski Resort. Emergency rescue is the central link of the whole ski resort safety rescue work. It refers to the ability to provide emergency help and professional medical aid to tourists in a timely manner after an accident. In order to improve the professional ability and business level of the emergency rescue of ski resorts and timely medical treatment in case of accidents, ski resorts should establish a long-term cooperative relationship with nearby hospitals [13].

2.1.6. Improving the Quality of Skiing Equipment. Improving the quality of ski resort equipment, expand the scale of ski resorts, pay attention to and replace ski equipment in a timely manner, and improve the competitiveness of their own ski resorts.

The safety factors for injuries encountered in ski resorts mainly include natural factors, facility factors, and human factors. Human factors play a large part in the factors affecting ski safety. Accidents caused by skiers themselves are on the rise every year, especially for beginners.

2.2. Network Node Structure Based on the Wireless Sensor. Wireless sensor networks are based on network nodes, which are mainly composed of four parts: data acquisition, processing, transmission, and power supply [14-17]. The front end uses different sensors to collect or detect the information that should be collected and monitored by the monitoring area. The data processing unit analyzes, processes, and stores data. The wireless module is responsible for the connection between remote components. The power supply is responsible for supplying power to the sensor assembly. This is the most basic set of wireless sensor components. Therefore, additional modules will be provided as needed [18], the structure of which is shown in Figure 1.

Compared with other networks, wireless sensor network has the advantages of low cost, multiredundant peer-to-peer network, high reliability, strong networking capability, multihop routing, etc., and is compatible with communication networks with various communication modes.

2.3. Perceived Network Structure. The perception network is to deploy nodes to the area that needs to be detected and use the principle that adjacent nodes can be connected to transmit data to build a huge wireless network. Each node monitors the data collected in the area in a collaborative or noncollaborative way, sends the information to the sink node through multiple transmissions, rotation, and other ways, and then sends the information to users through the sink node. The user then controls the node according to the transmitted data [19]. The architecture of the wireless sensor network is shown in Figure 2.

2.4. Characteristics of the Wireless Sensor Node Network. The perception network has the following characteristics:

(1) Self-organization: before setting up the network and sending data, there is no need to set up network equipment, and the site is automatically configured and managed. Once published, networks that can be controlled and collected can be quickly created through network processes and topology control systems [20].

(2) Dynamic network topology: wireless sensor nodes can be moved. Nodes may fail or lose power, resulting in various problems and exit from the network. Some nodes will be disconnected from the network from time to time, while new nodes are added to the network. This indicates that the network topology may be able to withstand the changes of the network system from time to time [21].

(3) Multihop routing: the data collection range of sensor components is only a few hundred meters, and the communication capability is very limited. To communicate with sensors outside the display area, the transmission must be controlled by intermediate nodes.

(4) Many nodes and wide distribution: the distribution area of sensors is generally very large, so the density of the distribution in this area is also very high. This makes the scale of the wireless sensor network larger [22-24]. The workflow of the wireless sensor is shown in Figure 3.

2.5. Requirements of the Deployed Wireless Sensor Network. The area of the ski resort is generally large, so the wireless sensor network needs to be deployed in a large area, and the cut data need to ensure real-time and continuous collection, 


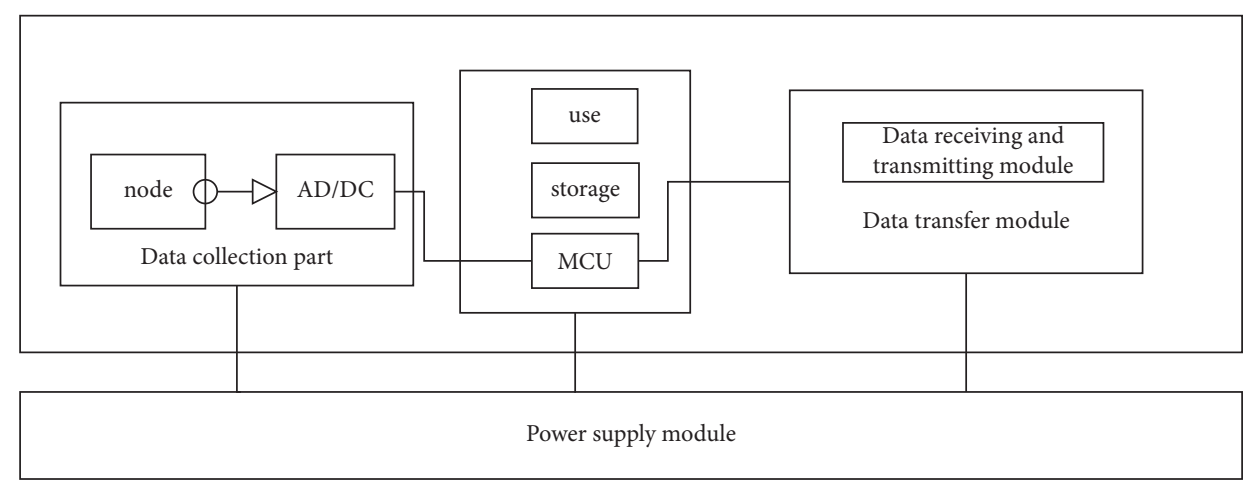

FIgURE 1: Structure diagram of the sensor network.

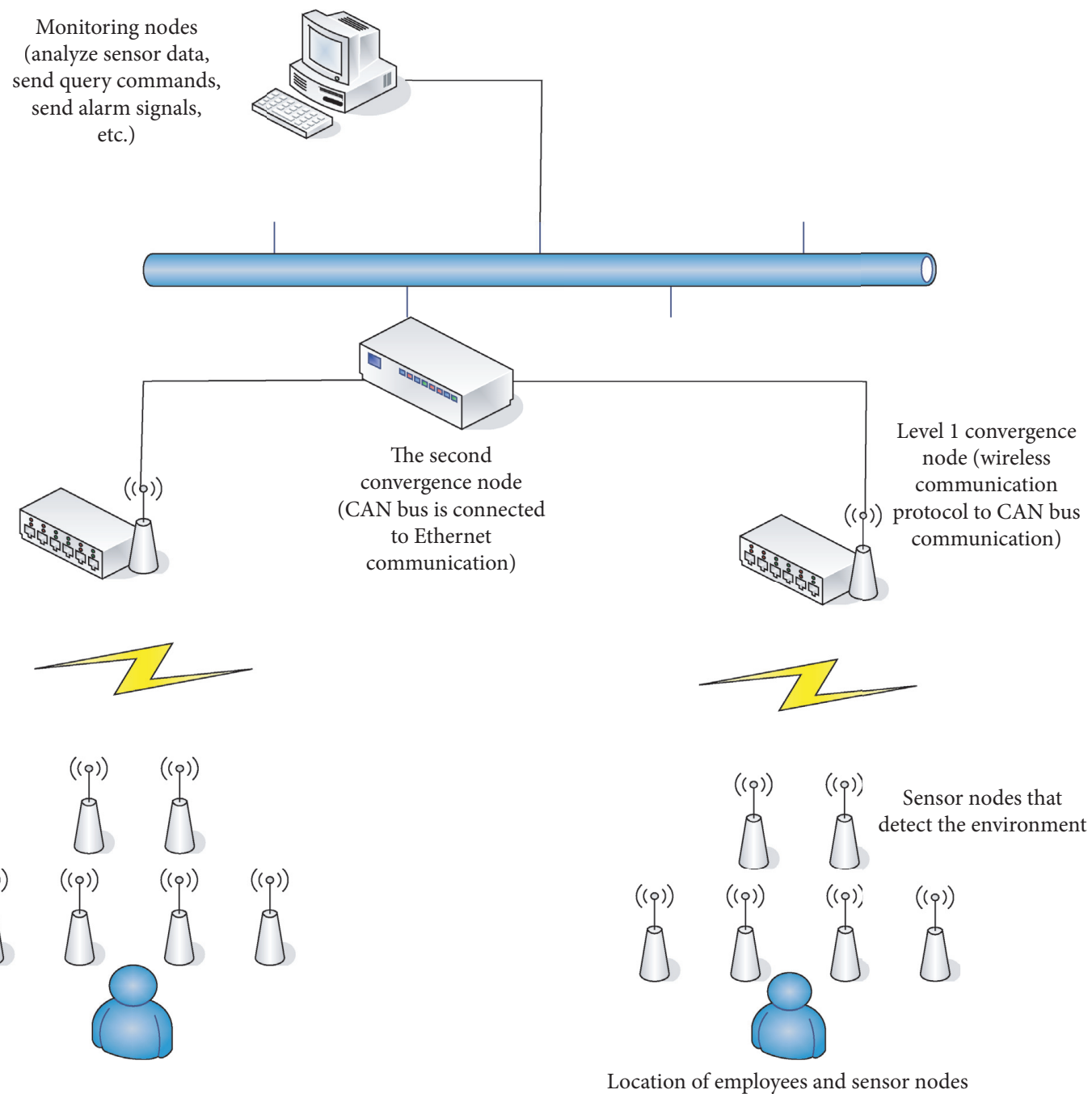

FIGURE 2: Architecture diagram of the wireless sensor network. 


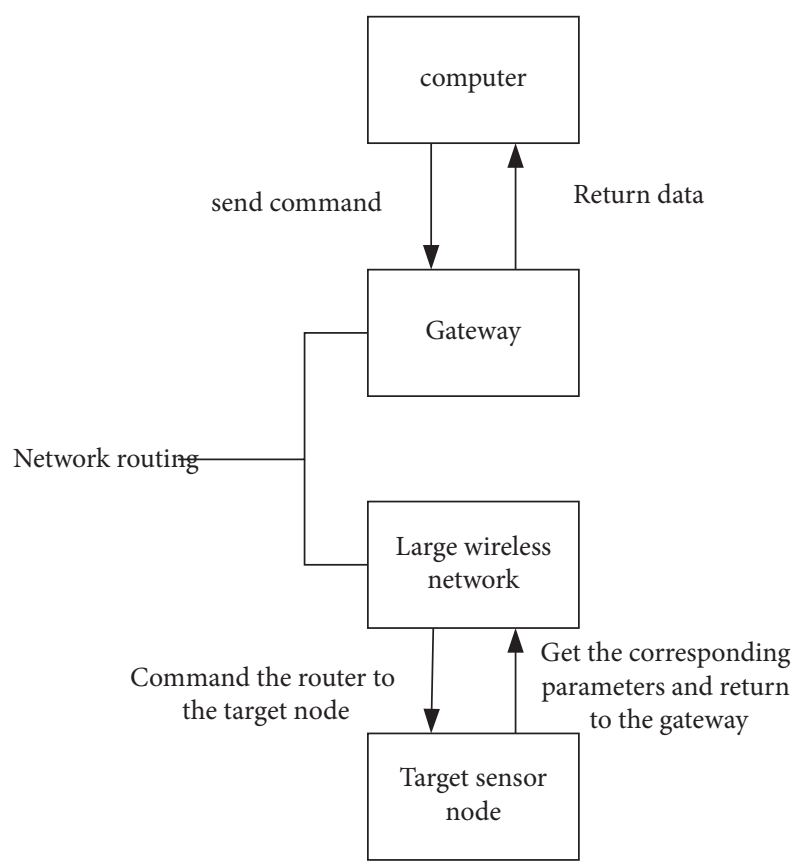

FIGURE 3: Flow chart of the wireless sensor.

processing, and aggregation. As an emerging technology in the twenty-first century, wireless perception networks can monitor and manage data in real-time [25]. All large-scale monitoring needs to be clear are as follows:

(1) Number of nodes

(2) The number of relay transmission nodes

(3) The frequency fluctuation of data collected by each node

(4) Maximum transmission power of the node

(5) The maximum power generated by node operation

(6) The maximum value of delayed data and the maximum allowable range of lost data

\subsubsection{Classification of Sensors and Wireless Communication} Technology. Sensors can be classified according to input, output, principle, basic effect, energy transformation, and technology, as shown in Figure 4.

Designing wireless sensor network nodes needs to follow the following main principles: (1) miniaturization and low cost; (2) low power consumption; (3) flexibility and scalability; (4) robustness.

Up to now, commonly used wireless communication technologies mainly include $\mathrm{Wi}-\mathrm{Fi}$, infrared, Bluetooth, and ZigBee. According to Table 1, the specific parameters of communication technology can be obtained as follows:

(1) Wi-Fi technology usually uses the IEEE $802.11 \mathrm{~b}$ standard, with the transmission rate reaching $11 \mathrm{Mbit} / \mathrm{s}$ and the communication distance reaching about 100 meters. Wi-Fi is a technology that connects devices to the Internet and is mostly applied to electronic devices such as laptops, mobile phones,

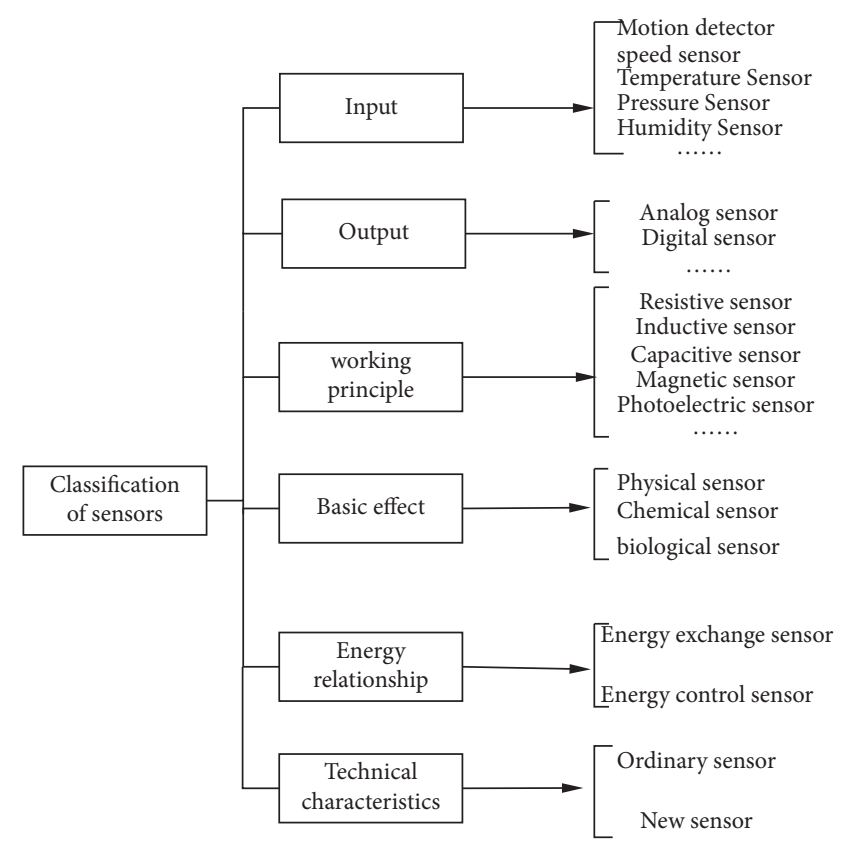

Figure 4: Sensor classification diagram.

and pads. However, due to high cost and power consumption, it cannot be popularized in wireless sensor networks [26].

(2) Infrared technology uses infrared light to transmit data information. Its implementation and operation are relatively simple and cost is low, but the infrared light is easily blocked, which requires no obstacles between devices and can only be directed transmission, so it cannot flexibly build a network.

(3) Bluetooth uses the IEEE 802.15.1 communication standard. The data transmission rate can reach $1 \mathrm{Mbit} / \mathrm{s}$, and the communication distance can reach $10 \mathrm{~m}$. Bluetooth is suitable for small amounts of data transmission. The typical application is wireless data communication between a mobile phone and a Bluetooth headset. Compared with ZigBee technology, its protocol is more complex, and node power consumption is high, so its application is limited to some extent.

(4) ZigBee technology adopts IEEE 802.15.4 standard. Compared with the above three communication technologies, it has the advantages of low power consumption, low cost, low complexity, and large network capacity, so it has a wide application and prospect in wireless sensor networks. The specific transmission rates are shown in Table 2.

Wireless sensor network (WSN) is a wireless data transmission network based on technical specifications and network protocols. Wireless sensor network has moderate transmission distance, reliable communication, avoiding problems caused by collisions, and short waiting time for transmission. This process is nonpatented, which greatly reduces the cost and enables wireless sensor networks to have automatic configuration, automatic 
TABle 1: Comparison table of the wireless communication technology.

\begin{tabular}{|c|c|c|c|c|}
\hline & Wi-Fi & IrDA & Bluetooth & ZigBee \\
\hline Frequency & $2.4 \mathrm{GHz}$ & Infrared & $2.4 \mathrm{GHz}$ & $2.4 \mathrm{GHz}$ \\
\hline Bandwidth & $11 \mathrm{Mbps}$ & $4 \mathrm{Mbps}$ & $1 \mathrm{Mbps}$ & $250 \mathrm{kbps}$ \\
\hline $\begin{array}{l}\text { Transmission } \\
\text { distance }\end{array}$ & $0-100 \mathrm{~m}$ & $\begin{array}{c}\text { Orientation } \\
1-2 \mathrm{~m}\end{array}$ & $0-10 \mathrm{~m}$ & $0-75 \mathrm{~m}$ \\
\hline Cost & High & Low & Lower & Lowest \\
\hline Number of linked & 30 & 2 & 7 & 65000 \\
\hline
\end{tabular}

Image and voice Small mobile Small data exchange, such as

Suitable for use in the field of automatic

Specific business data exchange device pictures and documents sensor control and various embedded devices

TABLE 2: Frequency bands and data transfer rates used by ZigBee technology.

\begin{tabular}{lcc}
\hline Working frequency & Data transfer rate & Number of channels \\
\hline $2.4 \mathrm{GHz}$ & $250 \mathrm{kbps}$ & 16 \\
$915 \mathrm{MHz}$ & $40 \mathrm{kbps}$ & 10 \\
$868 \mathrm{MHz}$ & $20 \mathrm{kbps}$ & 1 \\
\hline
\end{tabular}

recovery, and power management capabilities. A network can have multiple network nodes, and the entire network can support more nodes if assisted by the network coordinator. Combined with the integration of different network adapters, the number of nodes can be significant. It is very suitable for large-area sensor network layout. The specific sensor network structure is shown in Figure 5 .

\subsection{Use of Wireless Sensor Technology}

2.6.1. Compressed Sensing Technology. In the past few years, scientists have developed a technology that can compare sensing. This technology will not be limited by the Nyquist law and can construct a relatively perfect signal. The characteristics of CS technology include the integration of signal coding and sampling technology into perceptual compression technology. The distributed compression theory is an extension of CS. The biggest difference between DCS and CS is that CS receives one signal at a time while DCS can repeat multiple signals at the same time.

Compressed sensing technology mainly studies how to effectively capture and recover short signals from uncertain systems using a few observations. It should be noted that the compressed sensing model itself does not directly recognize $X$ signal but displays $X$ signal on the sensor panel to obtain the observed $Y$ value. The specific formula is as follows:

$$
Y=\Phi X
$$

Here, $X$ is an $\mathrm{n}$-dimensional discrete signal, namely, $X \in R^{N * N}$. If the signal is high-dimensional data, it can be vectorized. $Y$ is the observation vector, namely, $Y \in R^{M * 1}$; $\Phi \in R^{M * N}$ is the measurement matrix.

And, $K-$ signal $X$ is sparse $\Psi=\left\{\Psi_{i}\right\}_{i=1}^{N}$, i.e.,

$$
\begin{aligned}
X \sum_{i=1}^{N} \Psi_{i} S_{i} & =\Psi S, \\
\text { or } S & =\Psi^{-1} X .
\end{aligned}
$$

Here, $K$ represents the sparsity, and $K<N ; S$ is the transformation coefficient, and $S \in R^{N \times 1}$ is the other little column vectors. $\Psi$ is member of $\left\{\Psi_{1}, \Psi_{2}\right\} \in R^{N \times M}$ and represents transformation basis or sparse basis. $\Psi^{T}$ represents the transformation matrix, and $\Psi$ is the orthogonal matrix, so $\Psi^{T} \Psi=\Psi \Psi^{T}=I$ and $I$ in the formula represents the identity matrix.

Substitute (2) into (1) to obtain

$$
Y=\Phi X=\Phi \Psi S=\Theta S .
$$

In formula (3), $\Theta$ represents the data matrix of the sensor node, the holographic basis: $\Theta=\psi \Phi \in R^{M \times N}$.

In the process of perceptual compression, there are three parts as follows: signal sparsity, matrix design, and reconstruction algorithm design.

2.6.2. Selection of the Optimal Cluster Number. After completing network partitioning, the optimal number of clusters must be selected first, and then, the leading cluster must be selected. The number of cluster heads can directly affect the consumption when transferring data. If the number of clusters is large, the corresponding number of nodes will increase, consuming more energy in terms of data integration and gateway communication. However, if the number of clusters is small, the area covered by each cluster will increase, data will be transmitted through multiple jumps, and the amount of energy generated by data transmission will increase in proportion to the total energy. If the cluster size is too large, the lifetime of the whole network will be affected. Therefore, the optimal number of cluster heads must be analyzed to extend the service life of the entire wireless sensing network. To get the best number of cluster heads, LEACH energy consumption model is used to calculate.

In wireless sensor networks, the power gap between cluster nodes and other nodes is particularly obvious in data acquisition, transmission, and processing. The power model of the LEACH process is the output circuit, the power amplifier, and the receiving circuit. The power consumption of the amplifier is related to distance $d$, and the other two are 


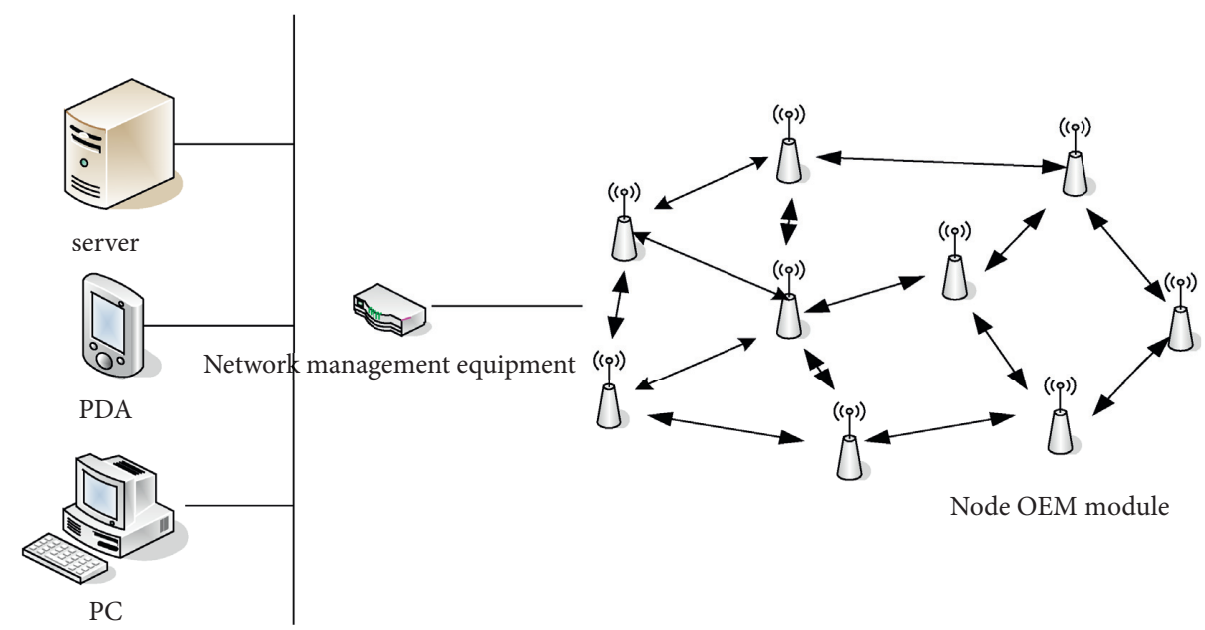

Figure 5: ZigBee wireless sensor network diagram.

related to the power consumption of the circuit itself. The specific formula for distance $d$ and energy consumption is as follows:

$$
d \begin{cases}d^{2}, & d<d_{0} \\ d^{4}, & d \geq d_{0}\end{cases}
$$

Here, $d_{0} \sqrt{e_{i s} / e_{m p}}, e_{m p}$, and $e_{i s}$ are all power attenuation coefficients. The former is for the multipath attenuation model, and the latter is for the free-space model. Based on the above information, the total energy consumption formula is as follows:

$E_{T}(L, d)=E_{t-\mathrm{elec}}(L)+E_{\mathrm{amp}}(L, d)= \begin{cases}L E_{\text {elec }}+L e_{f s} d^{2}, & d \leq d_{0}, \\ L E_{\text {elec }}+L e_{m p} d^{4}, & d>d_{0} .\end{cases}$

The power consumption formula for receiving Lbit information is as follows:

$$
E_{R}(L)=L E_{\text {elec }}
$$

$E_{\text {elec }}$ in formula (6) represents the energy consumed by the circuit when data are sent and received. The energy consumption formula of cluster head nodes in the first round is as follows:

$$
\begin{aligned}
E_{C H} & =E_{R}\left(\frac{n}{k}-1\right)+E_{D A} \frac{n}{k}+E_{T} \\
& =L\left(E_{\text {elec }} \frac{n}{k}+E_{D A} \frac{n}{k}+e_{m p} d_{t o B S}^{4}\right) .
\end{aligned}
$$

In formula (7), image represents the distance from any node to the NMS. Since the distance between the two is small, $d \propto d^{2}$ is used, where $d<d_{0}$. The formula of energy consumption of this node is as follows:

$$
E_{C M}=E_{T}=L\left(E_{\text {elec }}+e_{f s} d_{t o C H}^{2}\right) .
$$

$d_{\text {toCH }}$, in formula (8), is the distance between the node and the cluster. Since the sensor nodes are randomly placed, it is assumed that the density of $p_{i}$ is $p(x, y)$, and the coverage area of the partition is $m \times n$, so the expected distance formula is as follows:

$$
E\left[d_{t o C H}^{2}\right]=\iint r^{3} \times p(r, \theta) \times r \mathrm{~d} r \mathrm{~d} \theta .
$$

The radius of this region is $r=m / \sqrt{k \pi}$, which is substituted into formula (9) in combination with the function of distribution density to obtain

$E\left[d_{t o C H}^{2}\right]=\iint r^{3}+p \mathrm{~d} r \mathrm{~d} \theta=p \int_{0}^{2 \pi} \mathrm{d} \theta \int_{0}^{m / \sqrt{k \pi}} r^{3} \mathrm{~d} r=\frac{m^{2}}{2 k \pi}$.

By substituting formula (10) into formula (8), the energy consumption formula of each sensor node can be obtained as follows:

$$
E_{C M}=L\left(E_{\text {elec }}+\frac{e_{f s} m^{2}}{2 k \pi}\right)
$$

Combined with formula (11) and formula (7), the energy consumption of each cluster in a single operation can be obtained, as shown in the following formula:

$$
\begin{aligned}
E_{\text {cluster }} & =E_{C H}+\left(\frac{n}{k-1}\right) E_{C M} \approx E_{C H}+\left(\frac{n}{k}\right) E_{C M} \\
& =L\left[E_{\text {elec }}\left(\frac{2 n}{k}\right)+E_{D A}\left(\frac{n}{k}\right)+e_{e m} d_{t o B S}^{4}+e_{f s} \frac{m^{2} n}{2 k^{2} \pi}\right] .
\end{aligned}
$$

Then, formula (12) is used to obtain the total power consumed in the cycle.

$E_{\text {total }}=k E_{\text {cluster }}=L\left[E_{\text {elec }}(2 n)+E_{D A} n+k e_{m p}{\overline{d_{t o B S}}}^{4}+e_{f s} \frac{m^{2}}{2 k^{2} \pi}\right]$.

In formula (13), $\bar{d}_{t o B S}$ represents the average distance between the cluster head and the NMS. And the optimal number of clusters is as follows:

$$
\frac{\mathrm{d} E_{\text {total }}}{\mathrm{d} k}=l e_{m p} d_{t o B S}^{4}-l e_{f s}\left(\frac{m^{2}}{2 k^{2} \pi}\right) n=0 .
$$


Then, the formula to distinguish the optimal number of clusters is as follows:

$$
k_{\mathrm{opt}}=\sqrt{\frac{n}{2 \pi} \frac{e_{f s}}{e_{m p}} \frac{m}{\overline{d_{t o B S}}} .} .
$$

Figure 6 shows the flow of the LEACH algorithm.

In the security of traditional wireless sensor network, as the relay station of information forwarding, it is natural to choose the node with high trust. This leads to the excessive energy consumption of the node, which leads to network segmentation and other phenomena. Therefore, the trust degree of energy should be taken as reference data when building the model. The consumption formula of this node at runtime is as follows:

$$
\begin{aligned}
\text { Receiving_Cost }(k, d) & =E_{\text {elec }} \times k, \\
\text { Sending_Cost }(k, d) & =E_{\text {elec }} \times k+E_{\text {amp }} \times k \times d^{2} .
\end{aligned}
$$

Here, $Y$ and $K$, respectively, represent the transmission node and the number of data bits; $D$ represents the distance between the two nodes; and $E_{\text {elec }}$ and $E_{\text {amp, }}$, respectively, represent the energy consumption and the energy consumed by the transmission signal.

Therefore, if node $y$ transmits data according to a certain SNR, the energy consumption data are as follows:

$$
E C=2 \times \text { Eelec } \times k+\text { Eamp } \times k \times d^{2} .
$$

When the energy of the initial node is EB, the formula for the remaining energy ES when it passes through the $y$ node is as follows:

$$
E S=E B-E C \text {. }
$$

Therefore, when the energy trust value of the node reaches $E_{\text {th }}$, it means that the node can participate in the operation. Therefore, the trust degree ET $y$ of $y$ node is defined as follows:

$$
E T_{y} \begin{cases}1, & E S \geq E_{\mathrm{th}}, \\ 0, & E S \leq E_{\mathrm{th}} .\end{cases}
$$

\subsection{Sensor Routing Protocol Selection}

2.7.1. LEACH Routing Protocol. Because ski resort has the characteristics of large area, wide distribution, irregular shape, etc., the clustering routing protocol is chosen. For the special environment of the ski resort, the clustering routing protocol has the following advantages:

(1) Due to the large number of nodes distributed in the ski resort, it is difficult to maintain the nodes, so it is necessary to extend the network life. The clustering routing protocol can reduce the total energy consumption of the network and prolong the network life.

(2) In a bad environment, nodes are prone to accidental death. The clustering routing protocol divides the entire network into several clusters, and each cluster is independent of each other. When sensor nodes are

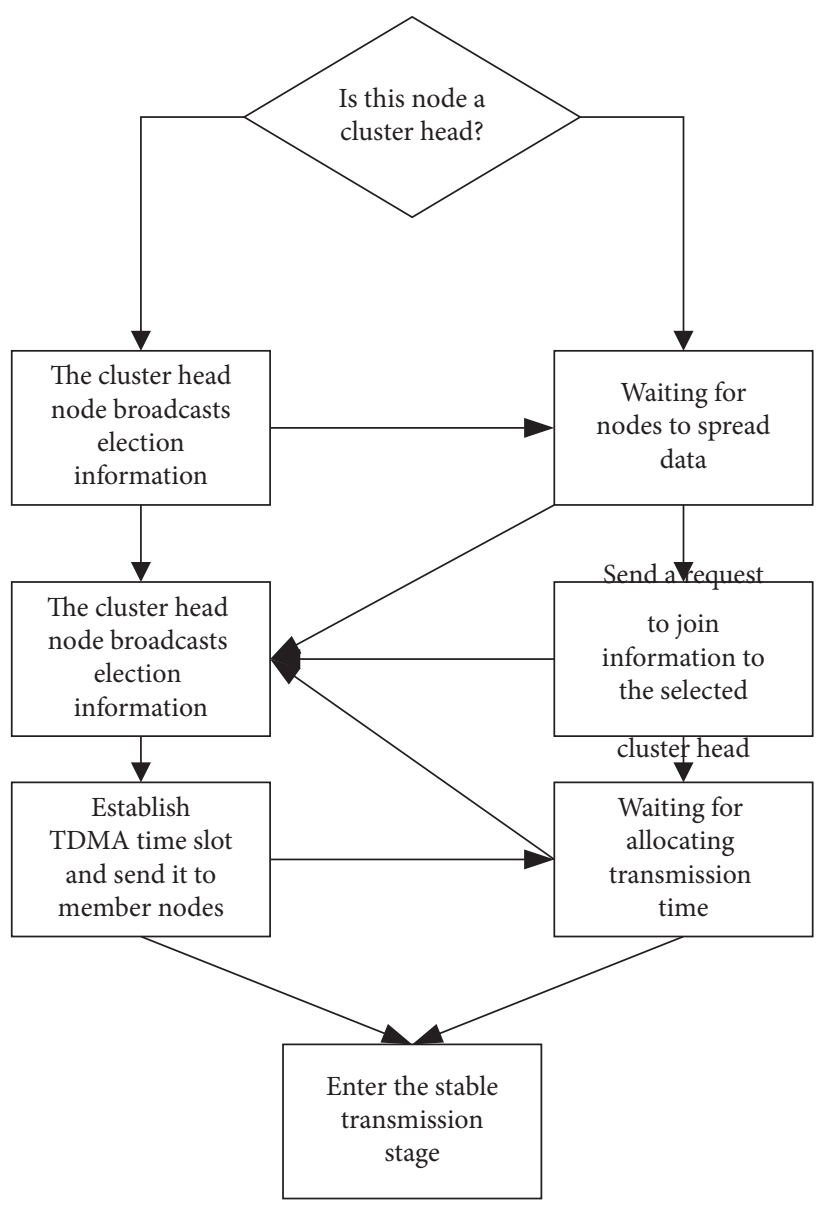

FIgUre 6: Flow chart of LEACH clustering algorithm.

disconnected from the network, the network topology changes little, so the network stability is very strong.

(3) The cluster-head node is responsible for transmitting the data of the network monitoring area and can also send the instructions conveyed by the monitoring platform to each node, which is very convenient for management.

2.7.2. LEACH-MH Routing Protocol. In view of the regional characteristics of the ski resort, a new energy-saving optimization routing algorithm is proposed based on LEACH protocol, which is named LEACH-MH. The LEACH protocol proposed earlier is a low power adaptive hierarchical routing algorithm in wireless sensor networks, but the nodes transmit data to the cluster head through one-hop communication, and the cluster head also transmits the aggregated data to the base station through one-hop communication. This will cause the cluster head node to be overloaded. The cluster head multihop algorithm is introduced in the LEACH protocol. The proposed LEACH-MH algorithm protocol makes a multihop optimal path between the cluster heads leading to the base station; thereby, reducing the energy consumption of the cluster head node prolongs the life of the sensor network and increases the 
TABle 3: Test data in the open area.

\begin{tabular}{|c|c|c|c|c|c|c|c|c|c|c|c|}
\hline Transmission distance $(\mathrm{m})$ & 10 & 50 & 100 & 150 & 200 & 250 & 300 & 350 & 400 & 450 & 500 \\
\hline Signal strength $(\mathrm{dBm})$ & -45 & -57 & -65 & -69 & -72 & -75 & -80 & -86 & -91 & -95 & -97 \\
\hline The amount of data packets sent & & & & & & 100 & & & & & \\
\hline Receive data volume & 100 & 100 & 100 & 99 & 98 & 96 & 94 & 92 & 90 & 54 & 8 \\
\hline Packet loss rate\% & 0 & 0 & 0 & 1 & 2 & 4 & 6 & 8 & 10 & 46 & 92 \\
\hline
\end{tabular}
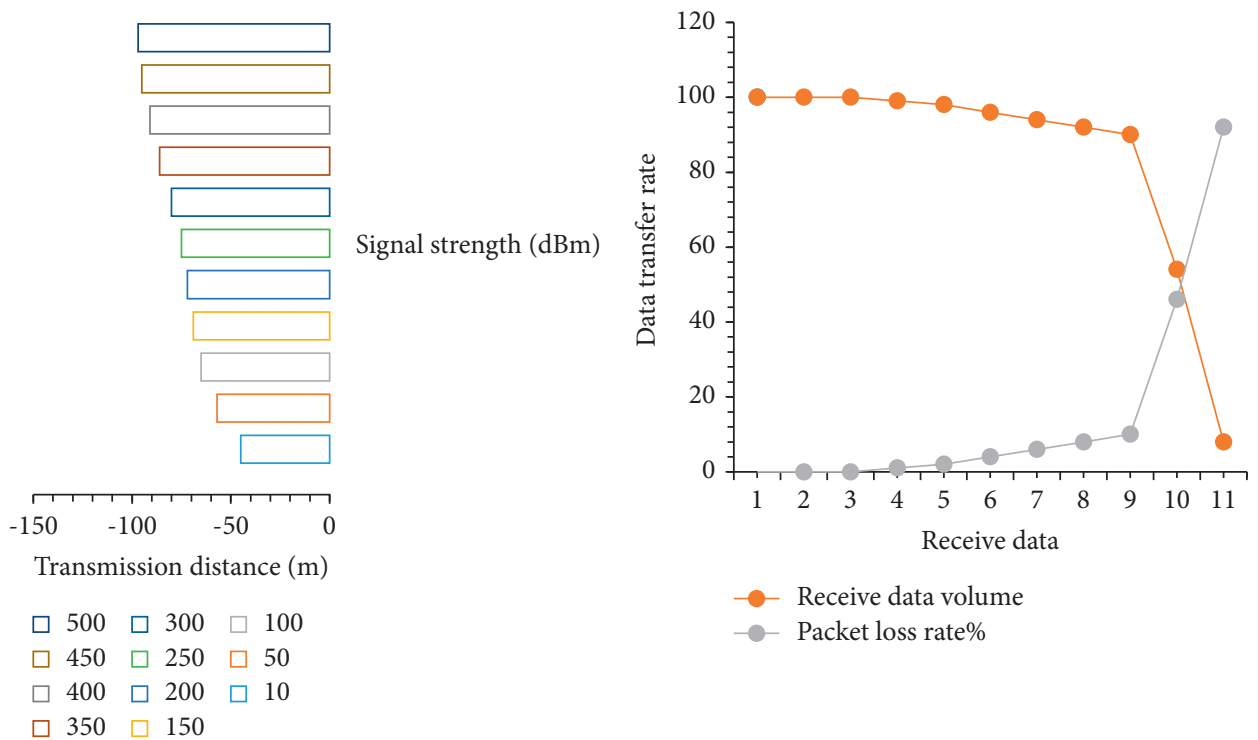

Figure 7: Open area test map.

TABLE 4: Transmission test in the snow forest area.

\begin{tabular}{|c|c|c|c|c|c|c|c|c|c|c|c|}
\hline Transmission distance $(\mathrm{m})$ & 1 & 5 & 10 & 20 & 30 & 40 & 50 & 55 & 60 & 65 & 70 \\
\hline Signal strength $(\mathrm{dBm})$ & -36 & -65 & -74 & -78 & -82 & -87 & -91 & -93 & -95 & -96 & -97 \\
\hline The amount of data packets sent & & & & & & 100 & & & & & \\
\hline Receive data volume & 100 & 100 & 100 & 99 & 96 & 92 & 90 & 81 & 54 & 23 & 8 \\
\hline Data loss rate\% & 0 & 0 & 0 & 1 & 4 & 8 & 10 & 19 & 46 & 77 & 92 \\
\hline
\end{tabular}

coverage of the network. Because in LEACH protocol, cluster communication is proportional to the square of distance squared. Therefore, the relay node should be selected as close to the cluster head as possible to be responsible for direct communication with the cluster head.

\section{Experiment Using the Wireless Sensor}

3.1. Wireless Sensor Transmission Test. In this experiment, three ZigBee modules were selected as coordinators, routers, and terminal nodes. Under the condition that the nodes are normal, the optimal transmission distance between the two nodes is tested in the open area and the forest area, respectively. As the distance between two nodes increases, the information data received by the wireless sensor are shown in Table 3.

As shown in Figure 7, the transmitted power of the node in this experiment is $35 \mathrm{dBm}$. The signal received strength is less than $97 \mathrm{dBm}$, and the data cannot be received, so the overall setting is above $97 \mathrm{dBm}$. According to the experimental data, the signal of the wireless sensing network in the open field is relatively stable within $400 \mathrm{M}$. When the distance exceeds $400 \mathrm{M}$, the issuer transmission begins to be unstable, the frequency of data loss begins to rise, and the data reception efficiency begins to drop rapidly.

As shown in Table 4, in the test based on snow forest, due to the coverage of trees and vegetation and the influence of rain and snow, the test distance is also different.

According to the data in Figure 8, the data obtained from the test in the woods of the ski resort show that the packet loss rate of the data is low when the distance is less than $50 \mathrm{~m}$. When the distance is larger than this, the packet loss rate increases dramatically, so the optimal communication distance in the forest is about $50 \mathrm{~m}$.

3.2. Comparison of Service Life of the Wireless Sensor. In this experiment, 100 nodes were selected to carry out experiments on the number of data transmission rounds in two protocols, at a distance of 50 meters and 100 meters, respectively.

As shown in Figure 9, the detection on the left is based on $50 \mathrm{M}$. As the number of test rounds continued, the first dead node of LEACH protocol appeared at about 175 rounds, while the node stopped running of LEACH-MH 

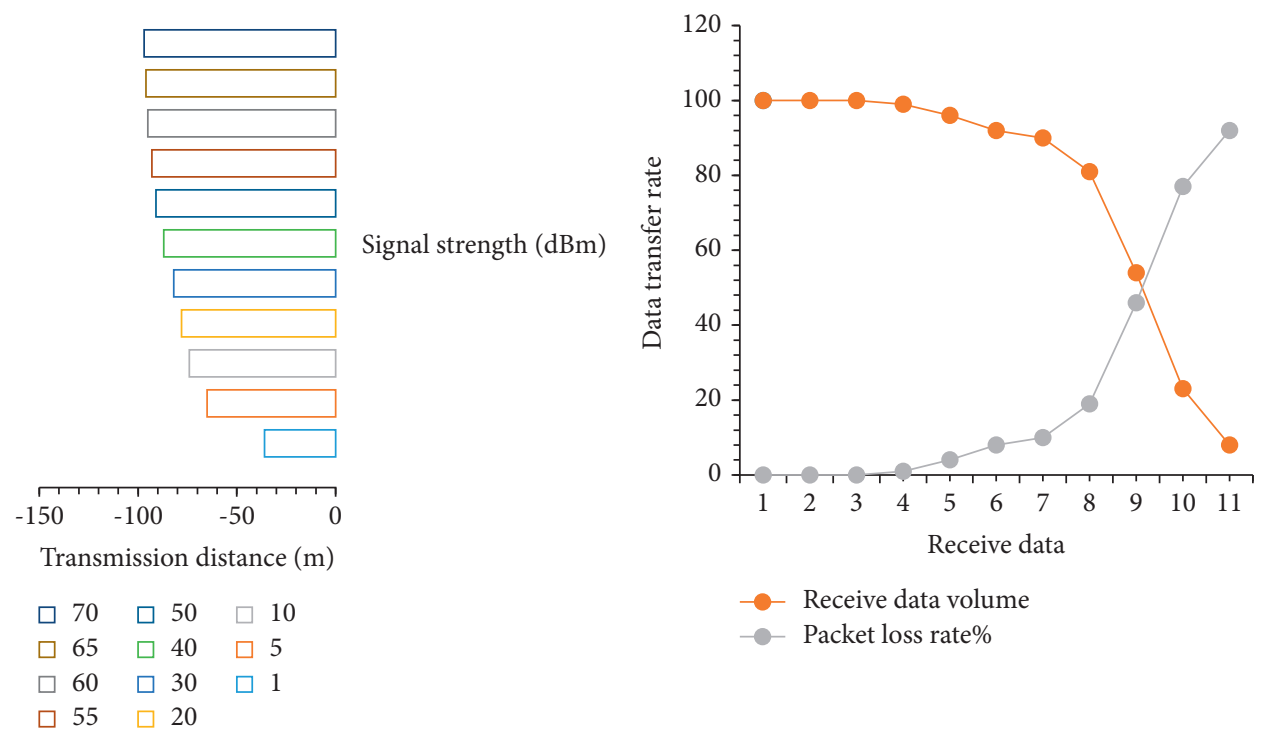

Figure 8: Data test chart of snow forest.
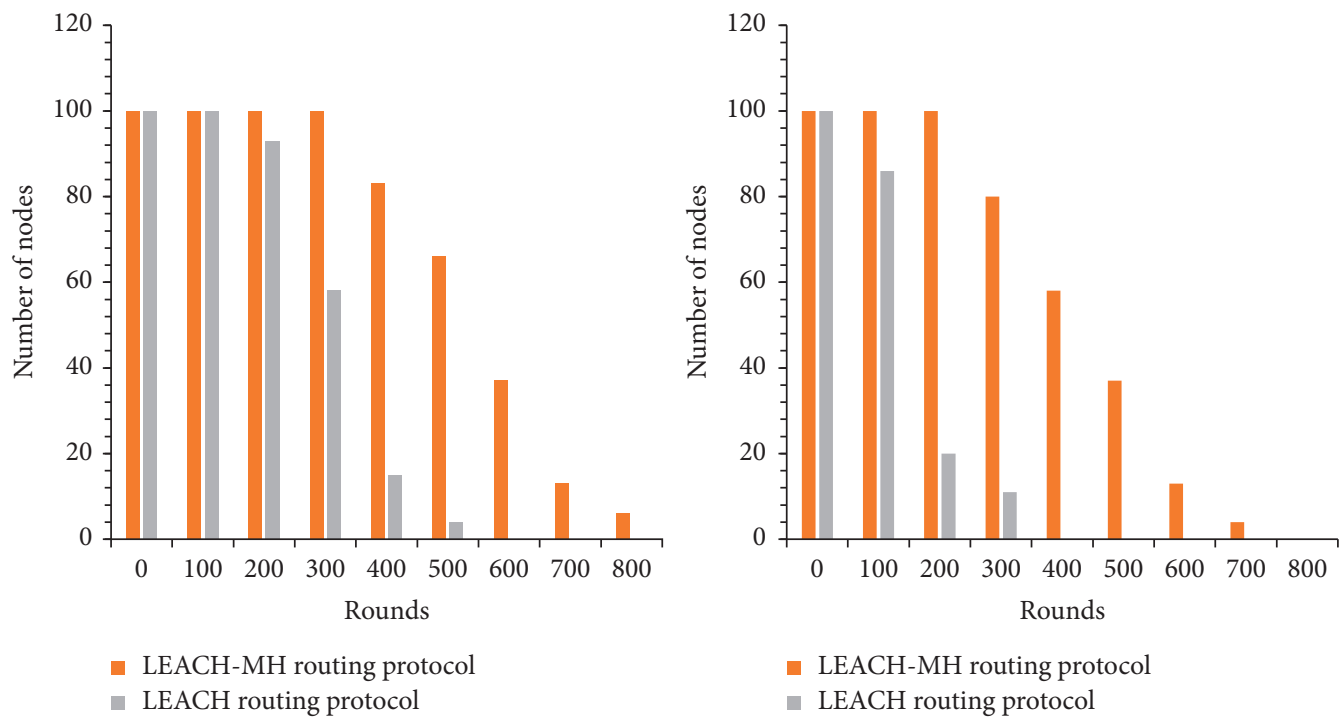

FIGURE 9: Change of the node number.

protocol appeared at about 360 rounds. In the test on the right based on the $100 \mathrm{~m}$ distance process, the node stopped running in the first 10 rounds of LEACH protocol. In $\mathrm{LEACH}-\mathrm{MH}$ protocol, the node stopped running around 190 rounds.

3.3. Comparison of Energy Loss. Energy-loss data represent an important indicator of wireless sensor operation. Figure 10 shows the data obtained from the energy test at a distance of $50 \mathrm{~m} * 50 \mathrm{~m}$ and $100 \mathrm{~m} * 100 \mathrm{~m}$.

As shown in Figure 10, at a scale of $50 \mathrm{M} * 50 \mathrm{~m}$, the total power consumed by the network reached 50J after running around 570 rounds in the test of LEACH protocol. On the basis of the LEACH-MH test, it still failed to reach 50J at 800 rounds. On the scale of $100 \mathrm{~m} * 100 \mathrm{~m}$ networks, 50J was achieved in only about 400 rounds of LEACH testing. In tests using the LEACH-MH protocol, it took 760 rounds to achieve a total energy consumption of 50J.

\section{Discussion of Wireless Sensor Network Experiments}

According to the data experiment in this paper, the influence of site on node distribution and network received data is tested, respectively, in the research on the distribution scale of wireless sensing network. According to the data, as the ski resort is large and partly covered by trees and vegetation, the optimal node distribution distance is $50 \mathrm{M}$, which can ensure the accuracy of data transmission. The data of the two routing protocols are compared on the basis of comparing the energy 

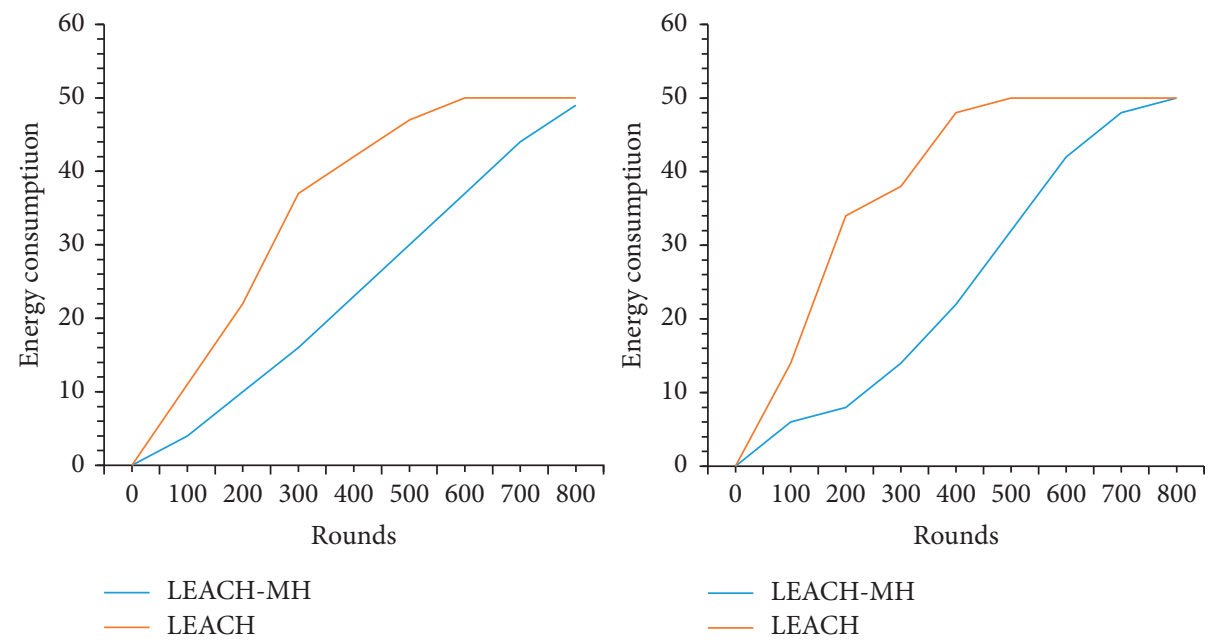

FIgURE 10: Energy consumption diagram.

consumption and lifetime of nodes. According to the data reality, the LEACH-MH protocol has more advantages than LEACH routing protocol, which can save energy and prolong life.

\section{Conclusion}

Based on the characteristics and advantages of the wireless sensor network, this paper makes full use of the latest wireless sensor network technology, such as sensor technology, wireless communication, and computer technology. This paper designs a training and detection system for the ski resort to ensure the safety of training personnel in the ski resort. In this paper, the original ski resort security system is analyzed and suggested, and then, the wireless network is reconstructed based on the wireless sensor. On the basis of the wireless sensor network of the new component, the factors affecting the network are analyzed, and then, the most suitable network routing protocol and network distribution scale are selected according to the data obtained from the experiment.

\section{Data Availability}

No data were used to support this study.

\section{Conflicts of Interest}

The authors declare no conflicts of interest.

\section{Authors' Contributions}

All authors have seen the manuscript and approved it for submission.

\section{Acknowledgments}

This work was supported under Grant no. 18TYB088.

\section{References}

[1] N. Abdul Latiff, M. Ruslee, S. Syed Yusof et al., "A training monitoring system for cyclist based on wireless sensor networks," Indonesian Journal of Electrical Engineering and Computer Science, vol. 6, no. 1, p. 80, 2017.

[2] C. Zhou, H. Luo, W. Fang, R. Wei, and L. Ding, "Cyberphysical-system-based safety monitoring for blind hoisting with the internet of things: a case study," Automation in Construction, vol. 97, pp. 138-150, 2019.

[3] L. Niu, W. Wang, and Y. Yu, "Design and research of wireless system for heavy metal monitoring based on optical sensor unit," Chinese Journal of Sensors \& Actuators, vol. 30, no. 3, pp. 433-437, 2017.

[4] A. S. Lutakamale and S. Kaijage, "Wildfire monitoring and detection system using wireless sensor network: a case study of Tanzania," Wireless Sensor Network, vol. 9, no. 8, pp. 274-289, 2017.

[5] X. Zhang, J. Du, C. Fan, D. Liu, J. Fang, and L. Wang, “A wireless sensor monitoring node based on automatic tracking solar-powered panel for paddy field environment," IEEE Internet of Things Journal, vol. 4, no. 5, pp. 1304-1311, 2017.

[6] M. Hammoudeh, F. Al-Fayez, and H. Lloyd, "A wireless sensor network border monitoring system: deployment issues and routing protocols," IEEE Sensors Journal, vol. 17, no. 8, p. 1, 2017.

[7] J. Wang, Y. Zhang, and J. Wang, "PWDGR: pair-wise directional geographical routing based on wireless sensor network," IEEE Internet of Things Journal, vol. 2, no. 1, pp. 14-22, 2017.

[8] K. Abe and M. Nozue, "Monitoring system of railway bridge piers and wireless sensor networks," Japanese Railway Engineering, vol. 57, no. 1, pp. 18-21, 2017.

[9] S. Puvaneshwari and Abirami, "Smart system for monitoring cardiac patients using wireless sensor networks," International Journal of Applied Engineering Research, vol. 13, no. 6 Pt.1, pp. 3144-3149, 2018.

[10] I. Almarashdeh, M. K. Alsmadi, and T. Farag, "Real-time elderly healthcare monitoring expert system using wireless sensor network," International Journal of Applied Engineering Research, vol. 13, no. 6, pp. 3517-3523, 2018.

[11] B. Singh, S. Urooj, S. Mishra, and S. Haldar, "Blood pressure monitoring system using wireless technologies," Procedia Computer Science, vol. 152, no. C, pp. 267-273, 2019. 
[12] J. Yang, J. Kwon, and B. Kim, "Wireless sensor network based real-time turbidity monitoring system for floating fish cage," Journal of Institute of Control, Robotics and Systems, vol. 24, no. 2, pp. 127-132, 2018.

[13] D. S. And and G. Maheshkumar, "Wireless MEMS-based accelerometer sensor system for structure vibration and post defamation monitoring," Advances in Computational Sciences and Technology, vol. 10, no. 11, pp. 3197-3204, 2017.

[14] F. Xiao, "Multi-sensor data fusion based on the belief divergence measure of evidences and the belief entropy," Information Fusion, vol. 46, pp. 23-32, 2019.

[15] I. Butun, P. Osterberg, and H. Song, "Security of the internet of things: vulnerabilities, attacks, and countermeasures," IEEE Communications Surveys \& Tutorials, vol. 22, no. 1, pp. 616-644, 2020.

[16] O. I. Khalaf and G. M. Abdulsahib, "Frequency estimation by the method of minimum mean squared error and $P$ value distributed in the wireless sensor network," Journal of Information Science and Engineering, vol. 35, no. 5, pp. 1099-1112, 2019.

[17] B. Yang and M. Yang, "Data-driven network layer security detection model and simulation for the internet of things based on an artificial immune system," Neural Computing and Applications, vol. 33, no. 2, pp. 655-666, 2021.

[18] Y. Xu, J. Li, and J. Zhou, "Real-time crack displacements monitoring system for reactor containment buildings with wireless sensor networks," Journal of Geomatics, vol. 42, no. 3, pp. 7-9 13, 2017.

[19] T. Alhmi Ed At, "Low-power environmental monitoring system for zigbee wireless sensor network," KSII Transactions on Internet and Information Systems, vol. 11, no. 10, pp. 4781-4803, 2017.

[20] M. Shitalnandkishorvitekar and M. Patil, "Automatic bridge monitoring system using wireless sensor network," IOSR Journal of Electronics and Communication Engineering, vol. 12, no. 6, pp. 29-33, 2017.

[21] K. Gomathy, "A hospital healthcare monitoring system using wireless sensor networks," International Journal of Applied Engineering Research, vol. 13, no. 2 Pt.2, pp. 1109-1119, 2018.

[22] L. Joris, F. Dupont, P. Laurent, P. Bellier, S. Stoukatch, and J.-M. Redoute, "An autonomous sigfox wireless sensor node for environmental monitoring," IEEE Sensors Letters, vol. 3, no. 7, pp. 01-04, 2019.

[23] L. Sun, Y. Zhao, W. Sun, and Z. Liu, "Study on supply chain strategy based on cost income model and multi-access edge computing under the background of the internet of things," Neural Computing and Applications, vol. 32, no. 19, pp. 15357-15368, 2020.

[24] A. Farouk, J. Batle, M. Elhoseny et al., "Robust general N user authentication scheme in a centralized quantum communication network via generalized GHZ states," Frontiers of Physics, vol. 13, no. 2, pp. 1-18, 2018.

[25] V. D. Ambeth Kumar, D. Elangovan, G. Gokul, J. Praveen Samuel, and V. D. Ashok Kumar, "Wireless sensing system for the welfare of sewer labourers," Healthcare Technology Letters, vol. 5, no. 4, pp. 107-112, 2018.

[26] J. Li, Y. Yang, H. Yan, C. Liu, L. Dong, and G. Wang, "Quasiomnidirectional wireless power transfer for a sensor system," IEEE Sensors Journal, vol. 20, no. 11, pp. 6148-6159, 2020. 